\title{
BOUNDEDNESS OF THE CALDERÓN-ZYGMUND SINGULAR INTEGRAL OPERATORS ON Ba SPACES
}

\author{
WEN-DONG CHANG
}

(Communicated by Palle E. T. Jorgensen)

\begin{abstract}
A condition for the boundedness of a Calderon-Zygmund operator on a new class of Banach function spaces is studied.
\end{abstract}

\section{INTRODUCTION}

A new class of function spaces, denoted by $\mathrm{Ba}$, was introduced by $\mathrm{X}$. X. Ding in [2]. It includes not only the standard Lebesgue integrable spaces, some familiar Orlicz spaces, Orlicz-Sobolev spaces, but also many useful new function spaces. In the past few years, many results have been obtained pertaining to Ba spaces and have been used in both classical analysis and other branches of mathematics. (See, for example, [2-5], [8].)

The main result of this paper is the proof of the boundedness of the CalderonZygmund singular integral operator (we will call it $\mathrm{C}-\mathrm{Z}$ operator), especially the boundedness of the well-known Riesz transformation in Ba spaces.

\section{THE BASIC IDEA OF Ba SPACES}

Let $B=\left\{B_{1}, B_{2}, \ldots, B_{m}, \ldots\right\} ; a=\left\{a_{1}, a_{2}, \ldots, a_{m}, \ldots\right\}$. Here, $B$ is a sequence of Banach function spaces, and $a$ is a sequence of non-negative real numbers. Moreover, let $\phi(z)=\sum_{m=1}^{\infty} a_{m} z^{m}$ be an entire function. For $f \in \bigcap_{m=1}^{\infty} B_{m}$, we form a power series as follows

$$
I(f, \alpha)=\sum_{m=1}^{\infty} a_{m}\|f\|_{B_{m}}^{m} \alpha^{m} .
$$

Here $\|\cdot\|_{B_{m}}$ stands for the norm in the $B_{m}$ space. Now denote by $R_{f}$ the radius of convergence of the series $I(f, \alpha)$ and by $\mathrm{Ba}$ the following measurable function set

$$
\mathrm{Ba}=\left(f \mid f \in \bigcap_{m=1}^{\infty} B_{m}, R_{f} \neq 0\right) .
$$

Received by the editors June 20, 1989 and, in revised form, July 24, 1989.

1980 Mathematics Subject Classification (1985 Revision). Primary 47B38, 44A15. 
The set $\mathrm{Ba}$ is proved to be a Banach space when we define the norm of an element $f \in \mathrm{Ba}$ by $\|f\|_{\mathrm{Ba}}=\inf _{\alpha>0}(1 / \alpha \mid I(f, \alpha) \leq 1)$. For a detailed explanation, see [2].

In this paper we will restrict the Banach spaces $B_{m}=L_{p_{m}}\left(\mathbf{R}^{n}\right), m=$ $1,2, \ldots$, and $p_{m}>1$. For simplicity, we will denote $\|\cdot\|_{\mathrm{Ba}}$ by $\|\cdot\|$ and $\|\cdot\|_{L_{p_{m}}}$ by $\|\cdot\|_{p_{m}}$.

\section{THE BOUNDEDNESS OF THE C-Z OPERATOR}

We consider the following $\mathrm{C}-\mathrm{Z}$ operator $T$ on $L_{p}\left(\mathbf{R}^{n}\right)$

$$
T(f)(x)=\lim _{\varepsilon \rightarrow 0} \int_{|y|>\varepsilon} K(y) f(x-y) d y,
$$

where $K(y)$ is a smooth function defined in $\mathbf{R}^{n}$ except at $x=0 . K(y)$ is also positively homomgeneous of order $-n$ and its integral mean on the unit sphere $S^{n-1}$ is zero.

It is well known that the operator $T$ is bounded on $\operatorname{Lp}\left(\mathbf{R}^{n}\right)$ and

$$
\|T(f)\|_{p} \leq A_{p}\|f\|_{p},
$$

where $A_{p}$ only depends on $p$ and the dimension $n$. After an easy computation, we see that $A_{p}$ is continuous in $p$ and

$$
A_{p}=O(1 /(p-1)) \text { as } p \rightarrow 1^{+} \text {and } A_{p}=O(p) \text { as } p \rightarrow \infty .
$$

See, for example, [1] and [7]. Now we prove

Theorem 1. If there exists two positive constants $\alpha$ and $\beta$ such that the $\mathrm{Ba}$ spaces satisfy the following condition

$$
1<\alpha<p_{m}<\beta \quad \text { for all } a_{m} \neq 0,
$$

then the $\mathrm{C}-\mathrm{Z}$ operator $T$ is bounded on the $\mathrm{Ba}$ spaces.

Proof. if $\alpha<p<\beta$ then $\exists K>0$ such that $A_{p} \leq K$ by the continuity. So if (3) is satisfied, we have $A_{p_{m}} \leq K, m=1,2, \ldots$. By definition of the Ba norm, we can easily see

$$
I(f, 1 /\|f\|)=\sum_{m=1}^{\infty} a_{m}\|f\|_{p_{m}}^{m} /\|f\|^{m} \leq 1, \quad \text { for } \forall f \in \mathrm{Ba},
$$

so we have the following estimates

$$
\begin{aligned}
I(T(f), 1 / K\|f\|) & =\sum a_{m}\|T(f)\|_{p_{m}}^{m} /(K\|f\|)^{m} \\
& \leq \sum a_{m} A_{p_{m}}^{m}\|f\|_{p_{m}}^{m} /(k\|f\|)^{m} \leq 1,
\end{aligned}
$$

therefore

$$
\|T(f)\|=\inf _{c>0}(1 / c \mid I(T(f), c) \leq 1) \leq K\|f\|, \quad \text { for } f \in \mathrm{Ba} .
$$

The theorem has been proved. 
People believe that the sufficient condition (3) for the boundedness of $\mathrm{C}-\mathrm{Z}$ operator is also necessary. But the proof of this needs a more careful estimate on the constants of the $L_{p}$ boundedness. We will prove this for a well-known special kind $\mathrm{C}-\mathrm{Z}$ operator, the Riesz transformation, in the next section.

\section{The Boundedness of $R_{j}$ IN Ba SPaCes}

Let $f \in L_{p}, 1<p<\infty$, the $n$-dimensional Riesz transformation of $f$ is defined by

$$
\left(R_{j} f\right)(x)=C_{n} \int_{\mathbf{R}^{n}}\left(x_{j}-y_{j}\right) /|x-y|^{n+1} f(y) d y, \quad j=1,2, \ldots, n,
$$

here

$$
C_{n}=\Gamma\left(\frac{n+1}{2}\right) /\left(\pi\left(\frac{n+1}{2}\right)\right) .
$$

For any positive number $l>0$, we define two regions $I(l)$ and $J(l)$ in $\mathbf{R}^{n}$,

$$
\begin{aligned}
& I(l)=\left\{x|| x \mid \leq l, x_{i}>0, i=1,2, \ldots, n\right\}, \\
& J(l)=\left\{x|| x \mid>2 l, 0<\theta_{j}<\pi / 4\right\},
\end{aligned}
$$

where $\theta_{j}$ is the angle between $\overrightarrow{o x}$ and the $j$ th axis. (See Figure 1 below.) Now we define the function $f_{l}(x)$ by

$$
f_{l}(x)= \begin{cases}1 & \text { if } x \in I(l) \\ 0 & \text { otherwise }\end{cases}
$$

\section{Lemma.}

$$
\| R_{j} f\left(l(x)\left\|_{p} \geq B(p)\right\| f_{l}(x) \|_{p} \quad \text { for } 1<p<2,\right.
$$

$B(p)$ is independent of $l$ and $B(p) \rightarrow \infty$ as $p \rightarrow 1^{+}$, and

$$
\left\|R_{j} f_{l}(x)\right\|_{p} \geq C(p)\left\|f_{l}(x)\right\|_{p} \quad \text { for } 2 \leq p<\infty,
$$

$C(p)$ is independent of $l$ and $C(p) \rightarrow \infty$ as $p \rightarrow+\infty$.

Note the relationship between $B(p), C(p)$, and the $A_{p}$ in (2). (Also see $[6,7]$.)

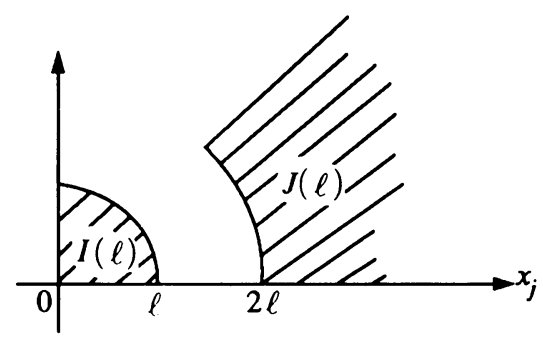

FIGURE 1 
Proof of the lemma.

$$
R_{j} f_{l}(x)=C_{n} \int_{\mathbf{R}^{n}}\left(x_{j}-y_{j}\right) /|x-y|^{n+1} f_{l}(y) d y=C_{n} \int_{I(l)}\left(x_{j}-y_{j}\right) /|x-y|^{n+1} d y .
$$

For $y \in I(l)$ and $x \in J(l), x_{j}-y_{j}+l \geq x_{j}$ and $x_{j} /|x|=\cos \theta_{j} \geq \cos \pi / 4=$ $\sqrt{2} / 2$, so we have $x_{j}-y_{j} \geq(\sqrt{2} / 2)|x|-l \geq(\sqrt{2} / 2)|x|-|x| / 2=(\sqrt{2} / 2-1 / 2)|x|$ and $|x-y| \leq x+l \leq 2|x|$, and so for any $x \in J(l)$

$$
\begin{aligned}
\left|R_{j} f_{l}(x)\right| & \geq\left[C_{n}(\sqrt{2}-1) / 2^{n+2}\right] \int_{I(l)} 1 /|x|^{n} d y \\
& =\left[C_{n}(\sqrt{2}-1) C l^{n} / n 2^{n+2}\right]|x|^{-n}
\end{aligned}
$$

the constant $C$ is defined by

$$
\int_{y \in I(l)} d y=C \int_{r=0}^{l} r^{n-1} d r=C l^{n} / n .
$$

Denoting the constant $C_{n}(\sqrt{2}-1) C / n 2^{n+2}$ by $C^{\prime}$, we have the following estimates,

$$
\begin{aligned}
\left\|R_{j} f_{l}(x)\right\|_{p} & \geq\left(\int_{x \in J(l)}\left|R_{j} f_{l}(x)\right|^{p} d x\right)^{1 / p} \\
& \geq C^{\prime} l^{n}\left(\int_{x \in J(l)}|x|^{-n p} d x\right)^{1 / p} \\
& =C^{\prime} l^{n}\left(C^{\prime \prime} \int_{r=2 l}^{\infty} r^{n(1-p)-1} d r\right)^{1 / p} \\
& =C^{\prime}\left(C^{\prime \prime}\right)^{1 / p} l^{n}\left[(2 l)^{n-n p} /(n p-n)\right]^{1 / p} \\
& =\left[C^{\prime}\left(C^{\prime \prime}\right)^{1 / p} 2^{(n-n p) / p} /(n p-n)^{1 / p}\right] l^{n / p} \\
& =\left[C^{\prime}\left(C^{\prime \prime}\right)^{1 / p} 2^{(n-n p) / p} n^{1 / p} / C^{1 / p}(n p-n)^{1 / p}\right]\left\|f_{l}\right\|_{p},
\end{aligned}
$$

where we have used $\left\|f_{l}\right\|_{p}=\left(C l^{n} / n\right)^{1 / p}$ and $C^{\prime \prime}$ is a constant independent of $l$ and $p$. If we let $B(p)=C^{\prime}\left(C^{\prime \prime}\right)^{1 / p} 2^{(n-n p) / p} n^{1 / p} / C^{1 / p}(n p-n)^{1 / p}$, then it is easy to see that $B(p) \rightarrow \infty$ as $p \rightarrow 1^{+}$, therefore (4) is proved.

It is easy to see that $\left\|R_{j} f_{l}\right\|_{p} /\left\|f_{l}\right\|$ is independent of $l$. By using a similar estimate, (5) can also be proved. But there is a simple way to see (5) is true. If it were not so, we would have a constant $k$ such that

$$
\left\|R_{j} f_{l}(x)\right\|_{p_{m}} \leq k\left\|f_{l}(x)\right\|_{p_{m}}, \quad m=1,2, \ldots, \quad \text { and } p_{m} \rightarrow \infty \quad \text { as } m \rightarrow \infty .
$$

Here $k$ is independent of $p$ and $l$. In particular $\left(\int_{I(l)}\left|R_{j} f_{l}\right|^{p_{m}} d x\right)^{1 / p_{m}}<$ $k\left\|f_{l}(x)\right\|_{p_{m}}$. Now letting $m \rightarrow \infty$, we have (note $\left.\left\|f_{l}(x)\right\|_{\infty}=1\right)\left|R_{j} f_{l}(x)\right| \leq k$ almost everywhere in $I(l)$, or $\left|\int_{I(l)}\left(x_{j}-y_{j}\right) /\right| x-\left.y\right|^{n+1} d y \mid \leq k$. Let $x \rightarrow 0$, we have $\int_{I(l)} y_{j} /|y|^{n+1} d y \leq k$. This is a contradiction since the integration is divergent. 
We are now ready to prove the main theorem.

Theorem 2. The Riesz transformation $R_{j} j=1,2 \ldots n$ are bounded operators in $\mathrm{Ba}$ spaces if and only if there exist constants $\alpha$ and $\beta$ such that (3) in $\S 3$ is satisfied.

Proof. The sufficiency has been proved in $\S 3$. We now prove the necessity of the condition (3).

We assume there exists a constant $A$, independent of $f$, such that

$$
\left\|R_{j} f\right\| \leq A\|f\| \quad \text { for all } f \in \mathrm{Ba} .
$$

By using (4) in the lemma and (7), we have

$$
\begin{aligned}
\sum_{m=1}^{\infty} & \left\{a_{m}\left(B\left(p_{m}\right)\left\|f_{l}(x)\right\|_{p_{m}}\right)^{m} /\left(A\left\|f_{l}\right\|\right)^{m}\right\} \\
& \leq \sum\left\{a_{m}\left(\left\|R_{j} f_{l}(x)\right\|_{p_{m}}\right)^{m} /\left\|R_{j} f_{l}\right\|^{m}\right\}=1 .
\end{aligned}
$$

In particular, $a_{m}^{1 / m} B\left(p_{m}\right)\left\|f_{l}\right\|_{p_{m}} /\left(A\left\|f_{l}\right\|\right) \leq 1$, or

$$
a_{m}^{1 / m}\left\|f_{l}\right\|_{p_{m}} /\left\|f_{l}\right\| \leq A / B\left(p_{m}\right) .
$$

Note that as $p \rightarrow 1+0, B(p) \rightarrow \infty$, so if $\alpha$ in (3) does not exist, we may find a $p_{m^{\prime}}>1$ such that

$$
a_{m}^{1 / m}\left\|f_{l}\right\|_{p_{m}} /\left\|f_{l}\right\|<\frac{1}{2} \quad \text { for } p_{m} \in\left(1, p_{m^{\prime}}\right] \text { and } l \in(0, \infty) .
$$

Without loss of generality, we assume there is $a_{m^{\prime \prime}}$ such that $a_{m^{\prime \prime}} \neq 0, p_{m^{\prime \prime}}<$ $p_{m^{\prime}}$, and

$$
0<a_{m^{\prime \prime}}^{1 / m^{\prime \prime}}\left\|f_{l}\right\|_{p_{m^{\prime \prime}}} /\left\|f_{l}\right\|<\frac{1}{2}
$$

Now choose $l_{0}$ large enough such that $C l_{0}^{n} / n>1$ and

$$
M\left(C l_{0}^{n} / n\right)^{1 / p_{m^{\prime}}}<a_{m^{\prime \prime}}^{1 / m^{\prime \prime}}\left(C l_{0}^{n} / n\right)^{1 / p_{m^{\prime \prime}},}
$$

where $M=\sup \left(a_{m}^{1 / m}, m=1,2, \ldots\right)<\infty, C$ is defined by (6). We can see for any $p_{m}>p_{m^{\prime}}$,

$$
a_{m}^{1 / m}\left(C l_{0}^{n} / n\right)^{1 / p_{m}} \leq M\left(C l_{0}^{n} / n\right)^{1 / p_{m^{\prime}}} \leq a_{m^{\prime \prime}}^{1 / m^{\prime \prime}}\left(C l_{0}^{n} / n\right)^{1 / p_{m^{\prime \prime}}},
$$

so, by using (10) and the fact $\left\|f_{l_{0}}\right\|_{p}=\left(C l_{0}^{n} / n\right)^{1 / p}$, we have

$$
a_{m}^{1 / m}\left\|f_{l_{0}}\right\|_{p_{m}} /\left\|f_{l_{0}}\right\| \leq a_{m^{\prime \prime}}^{1 / m^{\prime \prime}}\left\|f_{l_{0}}\right\|_{p_{m^{\prime \prime}}} /\left\|f_{l_{0}}\right\|<1 / 2 \quad P_{m}>P_{m^{\prime}} .
$$

(9) and (11) together give

$$
\sum_{m=1}^{\infty} a_{m}\left\|f_{l_{0}}\right\|_{p_{m}}^{m} /\left\|f_{l_{0}}\right\|^{m}<\sum_{m=1}^{\infty}\left(1 / 2^{m}\right)=1
$$


which is a contradiction to the definition of the Ba norm, since we can easily check that $I\left(f_{l_{0}}, 1 /\left\|f_{l_{0}}\right\|\right)=1$.

Now we proceed to prove the existence of the $\beta$ in the theorem. Using (5) in the lemma and (7), we obtain (by using similar estimates)

$$
\sum\left\{a_{m}\left(C\left(p_{m}\right)\left\|f_{l}\right\|_{p_{m}}\right)^{m} /\left(A\left\|f_{l}\right\|\right)^{m}\right\} \leq 1 .
$$

So $a_{m}^{1 / m}\left\|f_{l}\right\|_{p_{m}} /\left\|f_{l}\right\| \leq A / C\left(p_{m}\right)$. Note that as $p \rightarrow \infty, C(p) \rightarrow \infty$; so if $\beta$ does not exist, we can find $p_{m^{\prime}}$ large enough such that

$$
a_{m}^{1 / m}\left\|f_{l}\right\|_{p_{m}} /\left\|f_{l}\right\|<\frac{1}{2} \quad \text { for } p_{m} \in\left[p_{m^{\prime}}, \infty\right) \text { and } l \in(0, \infty) \text {. }
$$

Similarly, we may assume $\exists m^{\prime \prime}$ such that $p_{m^{\prime \prime}}>P_{m^{\prime}}$ and

$$
0<a_{m^{\prime \prime}}^{1 / m^{\prime \prime}}\left\|f_{l}\right\|_{p_{m^{\prime \prime}}} /\left\|f_{l}\right\|<\frac{1}{2} \text { for } l \in(0, \infty) .
$$

Choose $l_{1}$ small enough such that $C l_{1}^{n} / n<1$ and

$$
M\left(C l_{1}^{n} / n\right)^{1 / p_{m^{\prime}}}<a_{m^{\prime \prime}}^{1 / m^{\prime \prime}}\left(C l_{1}^{n} / n\right)^{1 / p_{m^{\prime \prime}}},
$$

then for any $p_{m}<p_{m^{\prime}}$,

$$
a_{m}^{1 / m}\left(C l_{1}^{n} / n\right)^{1 / p_{m}} \leq M\left(C l_{1}^{n} / n\right)^{1 / p_{m^{\prime}}}<a_{m^{\prime \prime}}^{1 / m^{\prime \prime}}\left(C l_{1}^{n} / n\right)^{1 / p_{m^{\prime \prime}}} .
$$

So, from (13), we have

$$
a_{m}^{1 / m}\left\|f_{l_{1}}\right\|_{p_{m}} /\left\|f_{l_{1}}\right\| \leq a_{m^{\prime \prime}}^{1 / m^{\prime \prime}}\left\|f_{l_{1}}\right\|_{p_{m^{\prime \prime}}} /\left\|f_{l_{1}}\right\|<\frac{1}{2} \quad \text { for } p_{m}<p_{m^{\prime}} .
$$

Equations (12) and (14) give

$$
\sum_{m=1}^{\infty} a_{m}\left\|f_{l_{1}}\right\|_{p_{m}}^{m} /\left\|f_{l_{1}}\right\|^{m}<1
$$

which is again a contradiction to the definition of the Ba norm, and the theorem has been proved.

\section{REFERENCES}

1. A. P. Calderón and A. Zygmund, Singular integral operators and differential equations, Amer. J. Math. 79 (1957), 901-921.

2. X. X. Ding and P. Z. Luo, Ba spaces and some estimates of Laplace operator, J. Systems Sci. Math. Sci. 1 (1) (1981), 9-33.

3. X. X. Ding, On a new class of function spaces, Kexue Tongbao 26 (1981), 973-976.

4. X. X. Ding, P. Luo, and Y. Li, The solvability of non-linear parabolic equations in Ba spaces, J. Central China Teachers College No. 2 (1985), 1-9.

5. Y. H. Dong et al, A new class of function spaces and the Hilbert transformation, J. Math. Anal. Appl. 108 (1985).

6. P. Koosis, Introduction to $H_{p}$ spaces, Cambridge University Press, New York, 1981.

7. E. M. Stein, Singular integrals and differentiability properties of functions, Princeton University Press, Princeton.

8. W. D. Zhang, The boundedness of some integral operators in a new class of function spaces, MR 87f \#47082.

Department of Mathematics, State University of New York, Buffalo, New York 14214-3093 